\title{
Numerical Study on Mass Sensitivity of Magnetoelastic Biosensors with Concentrated Mass Load under Different Resonance Modes
}

\author{
Kewei Zhang and Yuesheng Chai \\ School of Materials Science and Engineering, Taiyuan University of Science and Technology, Taiyuan 030024, China \\ Correspondence should be addressed to Kewei Zhang; drzkw@126.com
}

Received 22 March 2016; Revised 16 May 2016; Accepted 6 June 2016

Academic Editor: Andrea Cusano

Copyright (C) $2016 \mathrm{~K}$. Zhang and Y. Chai. This is an open access article distributed under the Creative Commons Attribution License, which permits unrestricted use, distribution, and reproduction in any medium, provided the original work is properly cited.

\begin{abstract}
Magnetoelastic biosensors are an important type of resonant mode based mass-sensing device, for which mass sensitivity is a critical parameter to evaluate their performance. In this work, the effect of concentrated mass position on mass sensitivity $\left(S_{m}\right)$ of a magnetoelastic sensor under different resonance modes was theoretically studied. The effect of magnitude of loading mass on mass sensitivity for the first resonance mode was also studied. The results indicated that mass sensitivity as the function of loading position for all resonance modes was consistent with the law of function $S_{m}=A \cos ^{2} t$. By comparing the mass sensitivity for the sensor attached with concentrated mass and uniform mass, it was found that mass sensitivity was linearly proportional to the sum of the squares of the displacement of each loading point. For the first resonance mode, when the loading position satisfied $0 \leq x_{c} / l<0.3$ or $0.7<x_{c} / l \leq 1$, mass sensitivity decreased with loading mass increasing. The opposite trend was observed when $0.3<x_{c} / l<0.5$ or $0.5<x_{c} / l \leq 0.7$. When the concentrated mass was loaded at the nodal point (i.e., $x_{c} / l=0.5$ ), mass sensitivity was always zero no matter how the loading mass changed.
\end{abstract}

\section{Introduction}

With the advantages of wireless in detection, easiness in operation, and low cost, magnetoelastic biosensors as an important type of acoustic wave devices have been widely used for the detection of pathogens like Listeria monocytogenes [1, 2], Escherichia coli [1, 2], Staphylococcus aureus [2], Salmonella typhimurium [2-7], Bacillus anthracis spores [7, 8], Staphylococcus epidermidis [9], and other chemicals $[10,11]$. A magnetoelastic biosensor is typically made of a freestanding rectangular magnetoelastic strip immobilized with bioreceptors on its surface. Due to magnetostriction effect, a magnetoelastic sensor can be excited to vibrate by applying an alternative magnetic field. The working principle of a magnetoelastic biosensor is based on the shift in resonance frequency due to the mass change caused by the specific binding of bioreceptor target on the sensor surface. Therefore, a magnetoelastic biosensor is actually a mass-detection device. For such a device, a key parameter to evaluate its performance is mass sensitivity $\left(S_{m}\right)$ which is defined as the change in the resonance frequency per unit mass load. Mass sensitivity can be well determined if the targets (i.e., mass load) are uniformly immobilized on the whole sensor surface [1]. This kind of immobilization mode has been widely used for magnetoelastic biosensors in various applications [1-11]. However, recent study has shown that such immobilization mode is not the best way to obtain the optimized mass sensitivity due to the location dependence of mass sensitivity [12-14].

Ramasamy et al. [12] experimentally found that the resonance frequency of a magnetoelastic sensor changed with the concentrated mass position. Based on the simulation results, equations for determining the maximum and minimum mass sensitivity were established. Li and Cheng [13] did the experiments for studying the resonance frequency of a magnetoelastic sensor attached with uniform mass load at different locations under different resonance modes. The experimental results indicated that the resonance frequency not only changed with mass load position but also changed 
TABLE 1: The properties of the sensor material and selected loading positions for this study $[15,16]$.

\begin{tabular}{lccc}
\hline & Symbol & Unit & Value \\
\hline Young's modulus & $E$ & $\mathrm{GPa}$ & 105 \\
Density & $\rho$ & $\mathrm{kg} / \mathrm{m}^{3}$ & $7.9 \times 10^{3}$ \\
Poisson's ratio & $v$ & - & 0.33 \\
Length & $l$ & $\mathrm{~mm}$ & 1 \\
Width & $w$ & $\mathrm{~mm}$ & 0.2 \\
Thickness & $h_{s}$ & $\mu \mathrm{m}$ & 15 \\
Loading position & $x_{c} / l$ & - & $0,0.05,0.1,0.15, \ldots, 1.0$ \\
\hline
\end{tabular}

with resonance modes. They also found that when the mass load was attached on the node of a magnetoelastic sensor, the resonance frequency did not change. That is, mass sensitivity is zero when mass is loaded on the node. In our previous work, a vibration governing equation of a magnetoelastic sensor attached with a concentrated mass for the first resonance mode was established based on mechanical vibration theory [14]. The node position, resonance frequency, and thus mass sensitivity with a given loading position were mathematically calculated. In this work, the behavior of mass sensitivity of a magnetoelastic sensor under higher resonance modes was further investigated and the magnitude of load mass on mass sensitivity was also studied aiming at in-depth understanding the fundamentals of mass sensitivity of a magnetoelastic biosensor.

\section{Materials and Methods}

Figure 1 shows a magnetoelastic biosensor with a concentrated mass attached on its surface. Here, the commercially available soft magnetic alloy Metglas ${ }^{\mathrm{TM}} 2826 \mathrm{MB}$ is selected as the sensor material and its material properties as well as the loading positions are listed in Table 1 . The sensor size is $1 \mathrm{~mm} \times 0.2 \mathrm{~mm} \times 15 \mu \mathrm{m}$ which is the same as that in the published paper regarding magnetoelastic biosensors [8]. The experimental results have proved that the magnetoelastic sensor of this size can work well for real biodetection.

\section{Theory and Calculation}

In real applications for biodetection, an alternative magnetic field is applied along the length direction of a magnetoelastic sensor, so that the sensor is driven to vibrate longitudinally (i.e., vibrating along its length direction). In this study, the sensor is assumed to vibrate longitudinally along $x$-axis, and its kinetic energy $(T)$ and potential energy $(V)$ can be expressed as follows:

$$
\begin{aligned}
T= & \frac{1}{2} \int_{0}^{l} \rho_{s} A_{s}\left(\frac{\partial u(x, t)}{\partial t}\right)^{2} d x \\
& +\frac{1}{2} m_{c}\left(\frac{\partial u(x, t)}{\partial t}\right)_{x=x_{c}}^{2}, \\
V= & \frac{1}{2} \int_{0}^{l} \frac{E}{1-\nu} A_{s}\left(\frac{\partial u(x, t)}{\partial x}\right)^{2} d x,
\end{aligned}
$$

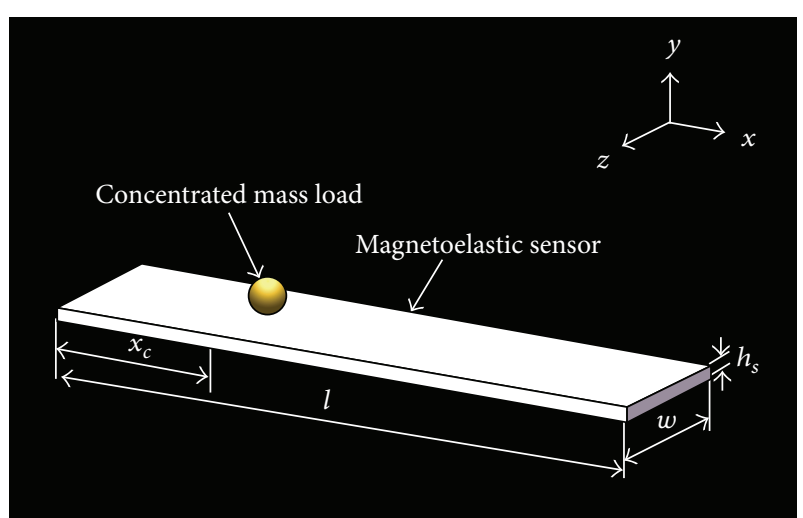

FIGURE 1: Schematic illustration of a magnetoelastic sensor with a concentrated mass loaded on its surface. $l, w$, and $h_{s}$ are the total length, width, and thickness of the sensor, respectively; the origin of coordinate is established at the middle of the left end of the sensor; $x$-axis is along the length direction; $y$-axis is along thickness direction; $z$-axis is along width direction; $x_{c}$ is $x$-coordinate of the concentrated mass load.

where $\rho_{s}, E, \nu$, and $A_{s}$ represent the density, Young's modulus, Poisson's ratio, and cross-sectional area $\left(w \times h_{s}\right)$ of the sensor, respectively, $m_{c}$ represents the loading mass, $\mathbf{u}(x, t)=$ $\boldsymbol{\varphi}(x) \mathbf{q}(t)$, where $\mathbf{u}(x, t)=\left[u_{1}(x, t), u_{2}(x, t), \ldots, u_{n}(x, t)\right]$, $u_{n}(x, t)$ is the displacement of point $x$ at time $t$ for the $n$ th-order resonance, $\varphi(x)=\left[\varphi_{1}(x), \varphi_{2}(x), \ldots, \varphi_{n}(x)\right]$, where $\varphi_{n}(x)$ is the mode shape function for the $n$ th-order resonance, $\mathbf{q}(t)$ is generalized coordinate which is a $n \times n$ matrix, and $u_{n}(x, t)=\varphi_{1}(x) q_{1 n}(t)+\varphi_{2}(x) q_{2 n}(t)+\cdots+$ $\varphi_{n}(x) q_{n n}(t)$. Theoretically, the potential energy $V$ in (2) consists of the potential energy of the sensor itself and that of biomolecules which depend on Young's modulus of the sensor material and biomolecules, respectively. Considering that Young's modulus of biomolecules is greatly less than that of the sensor material, the contribution of biomolecules to the potential energy is neglected in this study.

Equations (1) and (2) can be converted into

$$
\begin{aligned}
T & =\frac{1}{2} \dot{\mathbf{q}}^{T}\left(\mathbf{M}_{0}+\mathbf{M}_{1}\right) \dot{\mathbf{q}}=\frac{1}{2} \dot{\mathbf{q}}^{T} \mathbf{M} \dot{\mathbf{q}}, \\
V & =\frac{1}{2} \mathbf{q}^{T} \mathbf{K q},
\end{aligned}
$$

where $\mathbf{K}$ is the stiffness matrix in which the element $K_{i j}=$ $\int_{0}^{l}(E /(1-\nu)) A_{s} \varphi_{i}^{\prime}(x) \varphi_{j}^{\prime}(x) d x, \mathbf{M}_{0}$ is the mass matrix for the sensor in which the element $M_{0 i j}=\int_{0}^{l} \rho_{s} A_{s} \varphi_{i}(x) \varphi_{j}(x) d x, \mathbf{M}_{1}$ is the mass matrix for the concentrated mass in which the element $M_{1 i j}=m_{c} \varphi_{i}(x) \varphi_{j}(x)$, and $\mathbf{M}$ is the total mass matrix in which the element $M_{i j}=M_{0 i j}+M_{1 i j}$.

It is known that $\varphi_{n}=\cos (n \pi x / l)(n=1,2,3, \ldots)$ satisfies the boundary conditions of the sensor and thus $\varphi_{n}$ is selected as the shape functionforthe $n$ th-order resonance. 
By applying Lagrange's equation $(d / d t)(\partial L / \partial \dot{\mathbf{q}})-(\partial L / \partial \mathbf{q})=$ 0 (where $L=T-V$ ), the governing vibration equation of the sensor is derived as

$$
\left(\mathbf{K}-\omega_{n}^{2} \mathbf{M}\right) \mathbf{q}=\mathbf{0} .
$$

By solving (4), the generalized eigenvalue $\omega_{n}$ and $\mathbf{q}(t)$ are obtained. The $n$ th-order resonance frequency is then obtained by $f_{n}=\omega_{n} / 2 \pi$. The mass sensitivity $S_{m, n}$ for the $n$ thorder resonance is determined by

$$
S_{m, n}=\frac{f_{n, 0}-f_{n, m}}{m_{c}},
$$

where $f_{n, 0}$ and $f_{n, m}$ represent the $n$ th-order resonance frequency of the sensor without and with mass load. The point displacement for the $n$ th-order resonance is obtained by $u_{n}(x, t)=\varphi_{1}(x) q_{1 n}(t)+\varphi_{2}(x) q_{2 n}(t)+\cdots+\varphi_{n}(x) q_{n n}(t)$. All the calculations are done by MATLAB software.

\section{Results and Discussion}

From the above equations, we can see that the resonance frequency $f_{n}$ and mass sensitivity are dependent on the total mass matrix $\mathbf{M}$. Since $\mathbf{M}$ is the function of loading position $x$ and concentrated mass $m_{c}$, different loading positions and loading mass would result in different mass sensitivities. Figure 2(a) shows mass sensitivity $\left(S_{m}\right)$ as the function of loading position $\left(x_{c}\right)$ under different order resonance modes. Clearly, all the data curves exhibit periodic variation and there are $n+1$ loading positions (at $x / l=m / n$, where $n=1,2,3, \ldots$ and $m=0,1,2, \ldots, n)$ corresponding to the maximum $\left(S_{m, \max }\right)$ and $n$ loading positions (at $x / l=$ $(2 m-1) / 2 n$, where $n=1,2,3, \ldots$ and $m=1,2, \ldots, n)$ corresponding to the minimum $\left(S_{m}=0\right)$ for the $n$ th-order resonance. In addition, it is found that the data points in Figure 2(a) are well fitted by $S_{m}=A \cos ^{2} t$ ( $A$ is a constant) as presented by the dotted lines. That is, mass sensitivity is the function of only one independent parameter $t$ and is linearly proportional to $\cos ^{2} t$. Interestingly, it is found that $u^{2}$ as the function of $x_{c}$ as shown in Figure 2(b) shares the same variation trend as that of the curve in Figure 2(a) for the same order resonance mode. Therefore, it is believed that $|\cos t|$ is linearly proportional to $|u|$. In other words, $S_{m}$ is linearly proportional to $u^{2}$ at the point where the mass is loaded (i.e., $\left.S_{m} \propto u^{2}\right)$.

When the concentrated mass is uniformly loaded on the whole sensor surface, the mass sensitivity $\left(S_{m, u}\right)$ can be determined by the well-known equation [1]:

$$
S_{m, u} \approx \frac{f_{n}}{2 m_{s}},
$$

where $f_{n}$ is the $n$ th-order resonance frequency which is expressed as [15]

$$
f_{n}=\frac{n}{2 l} \sqrt{\frac{E}{\rho(1-\nu)}} .
$$

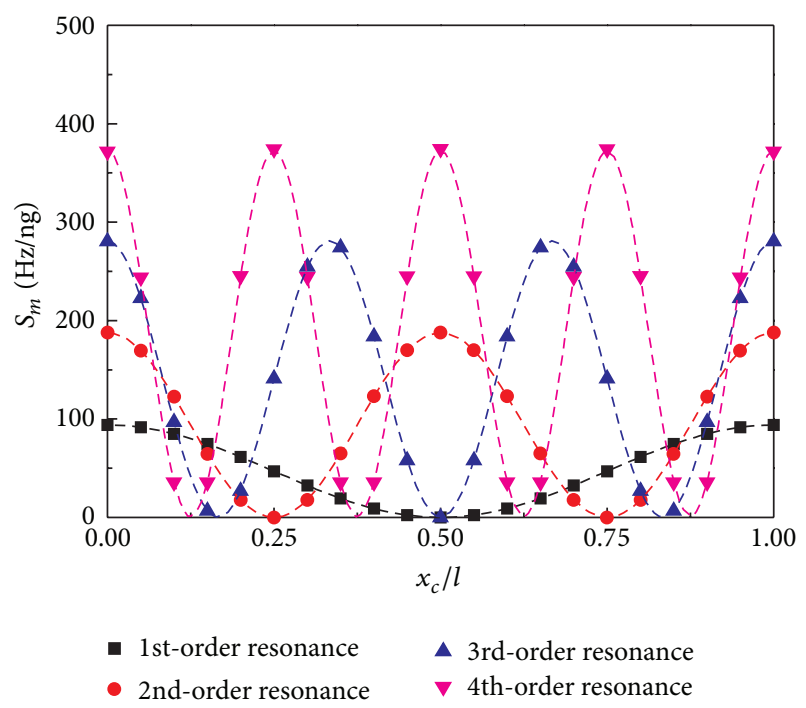

(a)

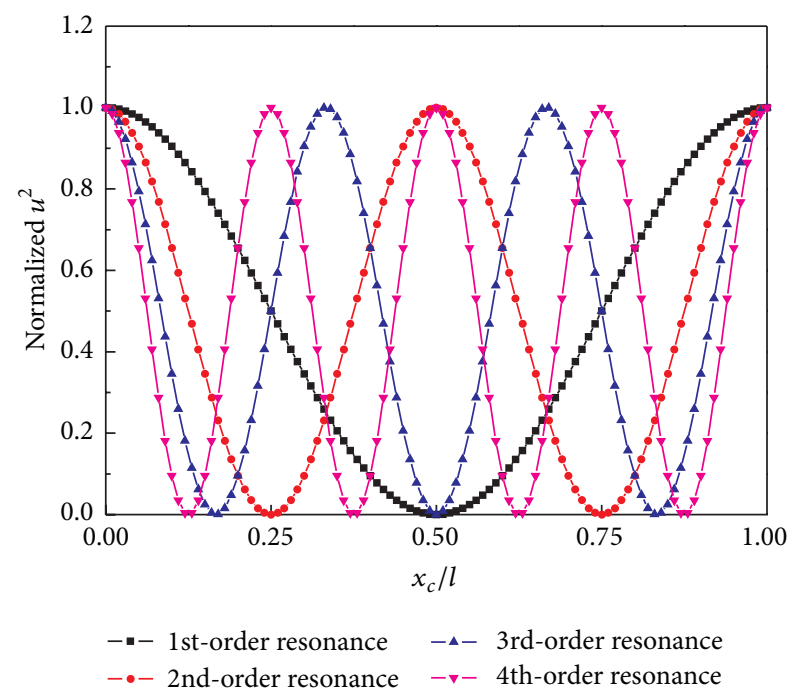

(b)

Figure 2: (a) Plot of mass sensitivity $\left(S_{m}\right)$ as the function of loading position; (b) plot of normalized square of point displacement as the function of position along $x$-axis; here $m_{c}=m_{s} / 1000$.

Figure 3 compares the plots of $S_{m, u}$ and the maximum mass sensitivity $S_{m, \max }$ in Figure 2(a) as the function of resonance order. Clearly, both curves show a linear relationship. In addition, it is found that $S_{m, \max } / S_{m, u}=2$ for the same resonance mode. Based on the aforementioned theory (i.e., $\left.S_{m} \propto u^{2}\right), S_{m, u}$ should be related to $u^{2}$ of all the points with mass load but we do not know what the relationship is. Now we assume the concentrated mass $\left(m_{c}=m_{s} / 1000\right)$ is evenly distributed on 102 positions (i.e., $x_{c} / l=0,0.01,0.02, \ldots, 1$ ) on the sensor. We find that $u_{\max }^{2} /\left(\sum_{i=1}^{101} u_{i}^{2} / 101\right) \approx 2$ and the value is very close to that of $S_{m, \max } / S_{m, u}=2$. Therefore, it is believed that mass sensitivity for uniform mass load is the average contribution of $u^{2}$ from all the loading points; that is, $S_{m, u} \propto \sum_{i=1}^{n} u_{i}^{2} / n$. 


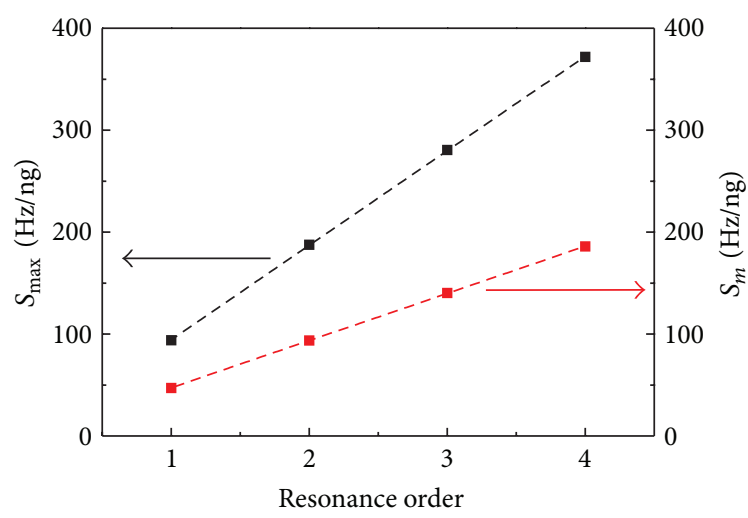

- Concentrated mass loading

- Uniform mass loading

FIgURE 3: Comparison of the maximum mass sensitivity in Figure 2(a) and mass sensitivity for uniform mass load as the function of resonance order.

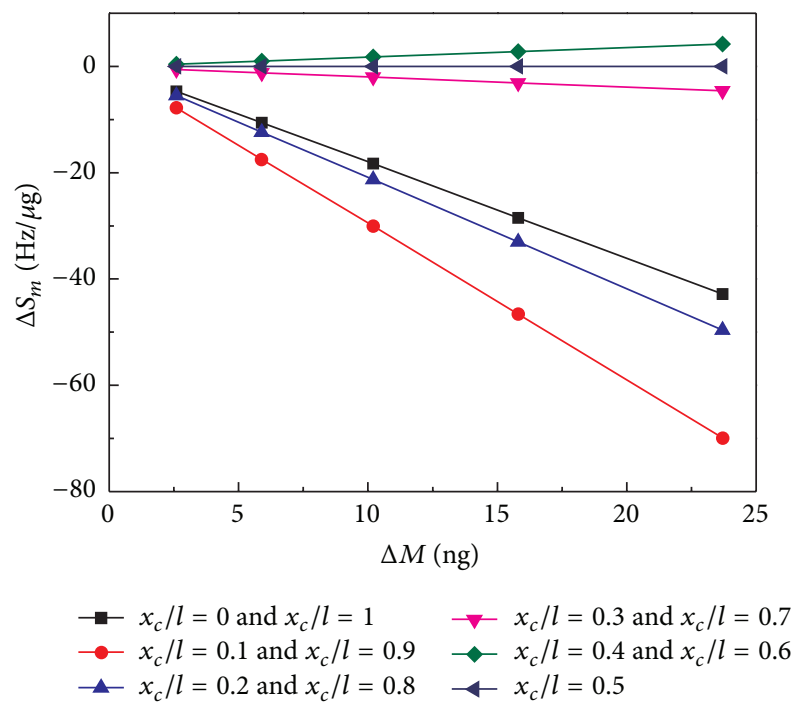

Figure 4: Plot of $\Delta S_{m}$ versus $\Delta M$ for different loading positions under the first-order resonance.

To investigate the effect of magnitude of mass load on mass sensitivity, mass sensitivities under six different loading masses (i.e., $m_{c 0}=m_{s} / 1000, m_{c 1}=m_{s} / 900, \ldots, m_{c 5}=$ $\left.m_{s} / 500\right)$ are calculated and compared. Here $S_{m 0}, S_{m 1}, \ldots, S_{m 5}$ are the mass sensitivities corresponding to the above six loading masses, respectively, and $\Delta S_{m}=S_{m, n}-S_{m, 0}$ and $\Delta M=m_{c, n}-m_{c 0}(n=1,2,3, \ldots, 5)$. Figure 4 shows the plot of $\Delta S_{m}$ as the function of $\Delta M$ for the first-order resonance.

It is found that $\Delta S_{m}$ can be either negative or positive or zero depending on the loading position. When the loading position satisfies $0 \leq x_{c} / l<0.3$ or $0.7<x_{c} / l \leq 1, \Delta S_{m}$ is negative and decreases as the loading mass increases which indicates that $S_{m}$ decreases with loading mass increasing. The opposite trend is observed when $0.3<x_{c} / l<0.5$ or $0.5<$ $x_{c} / l \leq 0.7$. When the concentrated mass is loaded at the nodal point (i.e., $x_{c} / l=0.5$ ), $\Delta S_{m}$ keeps zero no matter how the mass is changed. The above behaviors again can be confirmed

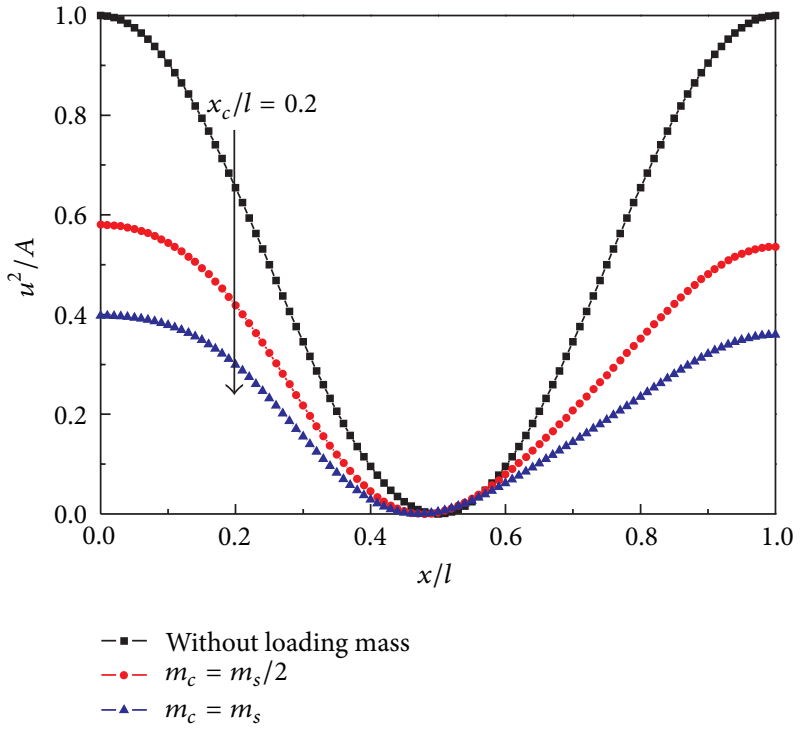

(a)

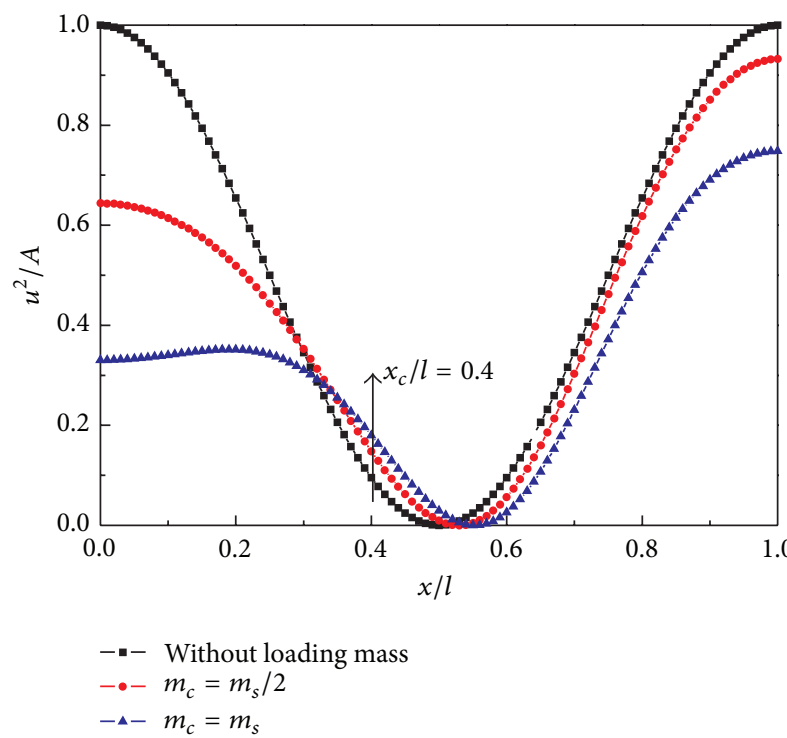

(b)

FIgURE 5: Plot of $u^{2} / A$ as the function of $x / l$ for the sensor with two different masses $\left(m_{c}=m_{s} / 2\right.$ and $\left.m_{c}=m_{s}\right)$ loaded at (a) $x / l=$ 0.2 and (b) $x / l=0.4$, respectively, under the first-order resonance. $A$ is a constant which represents the square of the maximum point displacement for the sensor without loading mass.

by $S_{m} \propto u^{2}$. Here we compare $u^{2}$ at $x / l=0.2\left(0 \leq x_{c} / l<0.3\right)$ and $x / l=0.4\left(0.3<x_{c} / l<0.5\right)$ where two different masses $\left(m_{c}=m_{s} / 2\right.$ and $\left.m_{c}=m_{s}\right)$ are loaded on the two positions, respectively, as shown in Figure 5. We can see that $u^{2}$ at the loading position $x_{c} / l=0.2$ decreases as $m_{c}$ increases while $u^{2}$ increases as $m_{c}$ increases at the loading position $x_{c} / l=0.4$ which agrees with $S_{m} \propto u^{2}$. For the mass loaded at the nodal point $\left(x_{c} / l=0.5\right)$, the plots of $u^{2}$ versus $x / l$ are exactly the same as that of the one for the sensor without loading mass. That is, $u^{2}$ at $x / l=0.5$ is always zero no matter how the load mass changes at $x / l=0.5$ which again is consistent with $S_{m} \propto$ $u^{2}$. 


\section{Implications}

The theoretical results provide important guidance on the immobilization patterns of bioreceptors and targets on a magnetoelastic sensor for biodetection, particularly for singletarget detection. For example, a single bacterial cell can be treated as a concentrated mass load. In such case, the position of the cell attached on the sensor would be very critical to mass sensitivity. In the worst case, that is, the cell attached on the node, there would be no frequency shift and thus a false result would be obtained. Based on the simulation results, we can conclude that the maximum mass sensitivity for the first resonance mode is obtained when the cell is attached on either end of the sensor. That means that the bioreceptor should be immobilized on the two ends of the sensor to ensure that the single target is attached on either end.

\section{Conclusion}

In this work, we established a governing equation of a magnetoelastic sensor attached with a concentrated mass under different resonance modes. Based on the results and analysis, several conclusions were drawn as follows:

(1) Mass sensitivity as the function of loading position $x_{c}$ can be well fitted by $S_{m}=A \cos ^{2} t$ and there are $n+1$ loading positions corresponding to the maximum and $n$ loading positions corresponding to the minimum $\left(S_{m}=0\right)$ for the $n$ th-order resonance.

(2) For the first resonance mode, when the mass load position $x_{c}$ satisfies $0 \leq x_{c} / l<\sim 0.3$ or $\sim 0.7<x_{c} / l \leq$ 1 , mass sensitivity decreases as loading mass increases while the opposite trend is obtained when $\sim 0.3<$ $x_{c} / l<\sim 0.5$ or $\sim 0.5<x_{c} / l \leq \sim 0.7$. Mass sensitivity does not change when concentrated mass is loaded at the nodal point $\left(x_{c} / l=0.5\right)$.

(3) Mass sensitivity comes from the average contribution of square of displacement $\left(u^{2}\right)$ of all the loading positions; that is, $S_{m, u} \propto \sum_{i=1}^{n} u_{i}^{2} / n$.

\section{Competing Interests}

The authors declare no conflict of interests.

\section{Acknowledgments}

This work was supported by the National Natural Science Foundation of China (Grant no. 51305290), the Shanxi Scholarship Council of China (Grant no. 2014-64), the Research Fund for the Doctoral Program of Higher Education of China (Grant no. 20131415120001), and Shanxi Province Science Foundation for Youths (Grant no. 2015021069).

\section{References}

[1] K. W. Zhang, L. Zhang, L. L. Fu, S. Q. Li, H. Q. Chen, and Z.-Y. Cheng, "Magnetostrictive resonators as sensors and actuators," Sensors and Actuators A: Physical, vol. 200, pp. 2-10, 2013.
[2] K. W. Zhang, L. L. Fu, L. Zhang, Z. Y. Cheng, and T. S. Huang, "Magnetostrictive particle based biosensors for in situ and real-time detection of pathogens in water," Biotechnology and Bioengineering, vol. 111, no. 11, pp. 2229-2238, 2014.

[3] M.-K. Park, S. Q. Li, and B. A. Chin, "Detection of Salmonella typhimurium grown directly on tomato surface using phagebased magnetoelastic biosensors," Food and Bioprocess Technology, vol. 6, no. 3, pp. 682-689, 2013.

[4] S. Q. Li, Y. G. Li, H. Q. Chen et al., "Direct detection of Salmonella typhimurium on fresh produce using phage-based magnetoelastic biosensors," Biosensors and Bioelectronics, vol. 26, no. 4, pp. 1313-1319, 2010.

[5] Y. T. Chai, S. Q. Li, S. Horikawa, M.-K. Park, V. Vodyanoy, and B. A. Chin, "Rapid and sensitive detection of Salmonella typhimurium on eggshells by using wireless biosensors," Journal of Food Protection, vol. 75, no. 4, pp. 631-636, 2012.

[6] M.-K. Park, J. W. Park, H. C. Wikle III, and B. A. Chin, "Evaluation of phage-based magnetoelastic biosensors for direct detection of Salmonella Typhimurium on spinach leaves," Sensors and Actuators B: Chemical, vol. 176, pp. 1134-1140, 2013.

[7] S. C. Huang, H. Yang, R. S. Lakshmanan et al., "Sequential detection of Salmonella typhimurium and Bacillus anthracis spores using magnetoelastic biosensors," Biosensors and Bioelectronics, vol. 24, no. 6, pp. 1730-1736, 2009.

[8] W. Shen, R. S. Lakshmanan, L. C. Mathison, V. A. Petrenko, and B. A. Chin, "Phage coated magnetoelastic micro-biosensors for real-time detection of Bacillus anthracis spores," Sensors and Actuators B: Chemical, vol. 137, no. 2, pp. 501-506, 2009.

[9] S. J. Huang, Y. J. Wang, S. T. Ge, Q. Y. Cai, and C. A. Grimes, "Quantification of Staphylococcus epidermidis using a wireless, mass-responsive sensor," Sensors and Actuators B: Chemical, vol. 150, no. 1, pp. 412-416, 2010.

[10] C. M. Ruan, K. F. Zeng, O. K. Varghese, and C. A. Grimes, "A staphylococcal enterotoxin B magnetoelastic immunosensor," Biosensors and Bioelectronics, vol. 20, no. 3, pp. 585-591, 2004.

[11] S. H. Wu, X. J. Gao, Q. Y. Cai, and C. A. Grimes, "A wireless magnetoelastic biosensor for convenient and sensitive detection of acid phosphatase," Sensors and Actuators B: Chemical, vol. 123, no. 2, pp. 856-859, 2007.

[12] M. Ramasamy, C. Liang, and B. C. Prorok, "Magnetomechanical MEMS sensors for bio-detection," in MEMS and Nanotechnology, Volume 2: Proceedings of the 2010 Annual Conference on Experimental and Applied Mechanics, Conference Proceedings of the Society for Experimental Mechanics Series, pp. 9-15, Springer, Berlin, Germany, 2011.

[13] S. Q. Li and Z.-Y. Cheng, "Nonuniform mass detection using magnetostrictive biosensors operating under multiple harmonic resonance modes," Journal of Applied Physics, vol. 107, no. 11, Article ID 114514, pp. 1-6, 2010.

[14] K. W. Zhang, Y. S. Chai, and Z.-Y. Cheng, "Location dependence of mass sensitivity for acoustic wave devices," Sensors, vol. 15, no. 9, pp. 24585-24594, 2015.

[15] C. Liang, S. Morshed, and B. C. Prorok, "Correction for longitudinal mode vibration in thin slender beams," Applied Physics Letters, vol. 90, no. 22, Article ID 221912, pp. 1-3, 2007.

[16] http://www.metglas.com. 


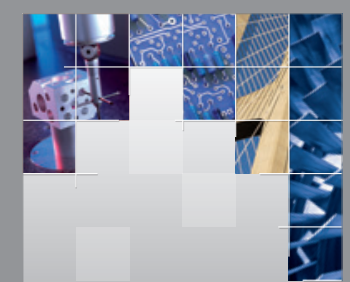

\section{Enfincering}
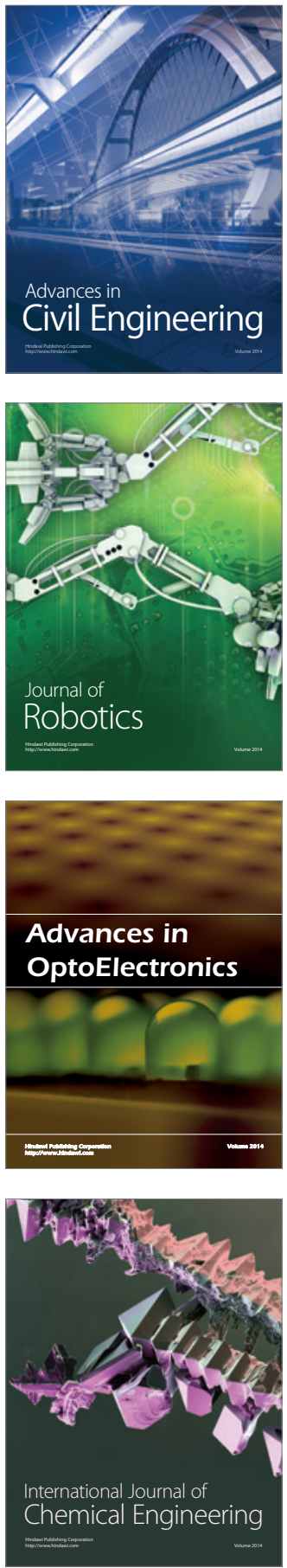

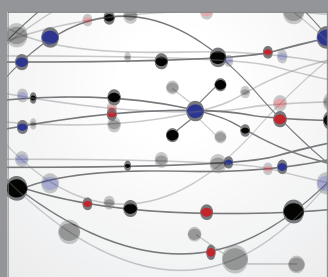

The Scientific World Journal

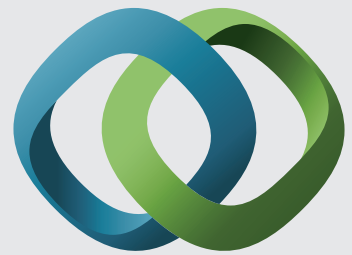

\section{Hindawi}

Submit your manuscripts at

http://www.hindawi.com
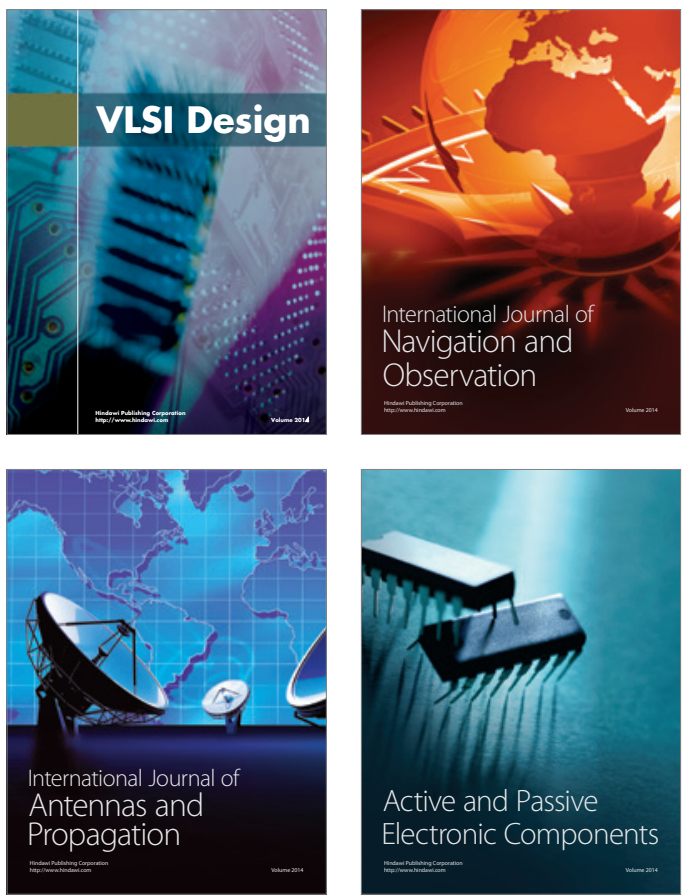
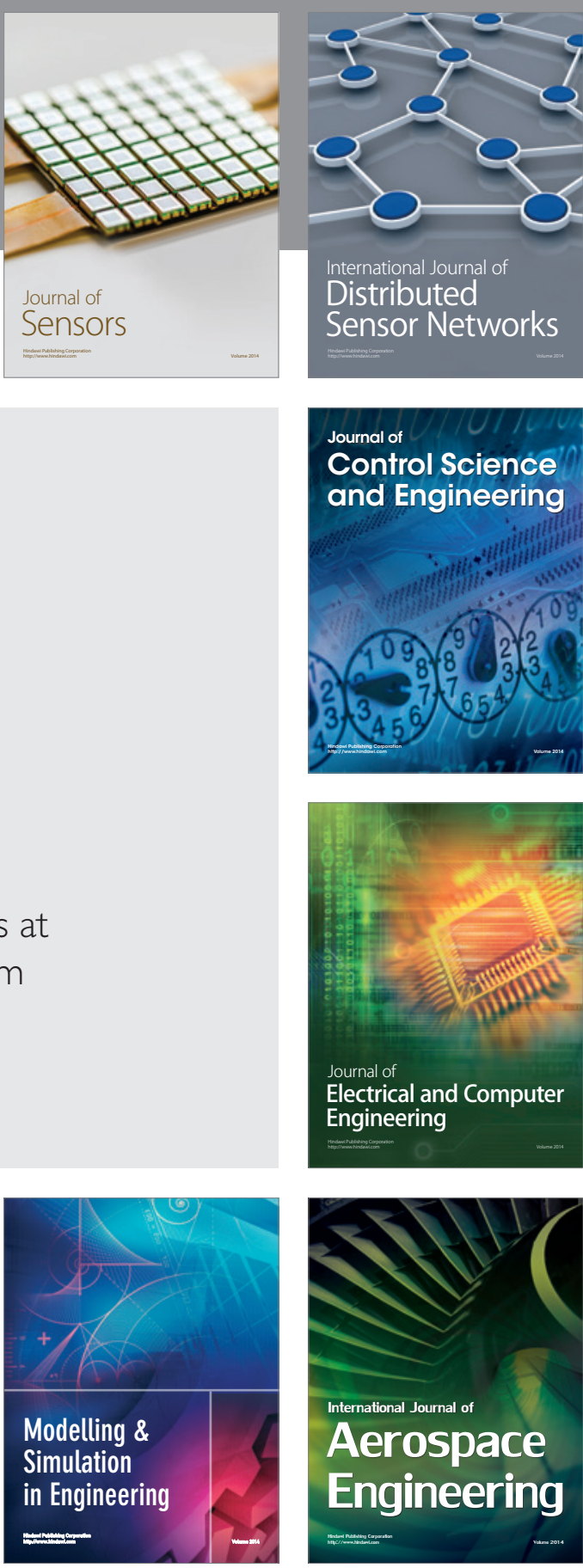

International Journal of

Distributed

Sensor Networks

Journal of

Control Science

and Engineering
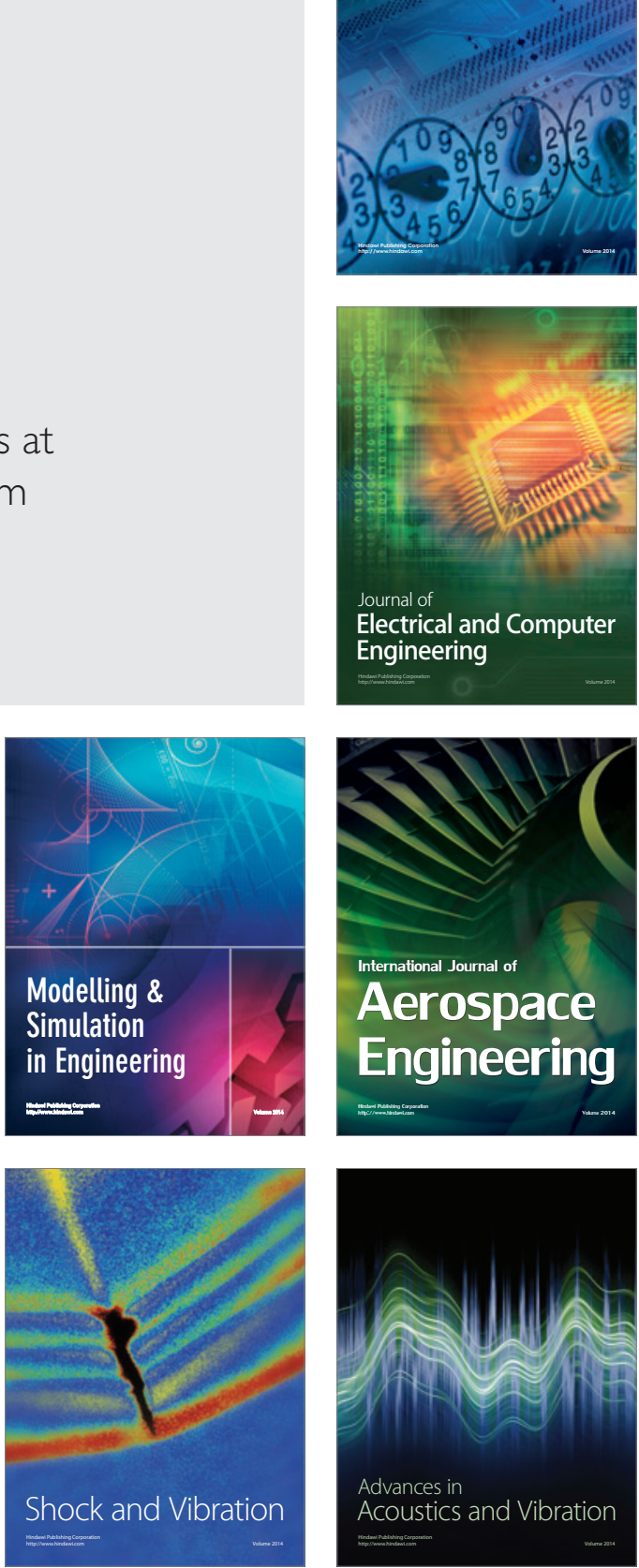